\title{
IMPROVEMENT OF FAULT DETECTION METHOD \\ FOR NONLINEAR BLACK-BOX SYSTEMS BASED ON MULTI-FORM QUASI-ARMAX MODELING
}

\author{
K.Kumamaru ${ }^{*}$ K.Inoue ${ }^{*}$ F.Tsubouchi ${ }^{*}$ and T.Söderström ${ }^{* *}$ \\ * Faculty of Computer Science and Systems Engineering, Kyushu \\ Institute of Technology Kawazu 680-4, Iizuka 820-8502, \\ Fukuoka, Japan e-mail:kumamaru@ces.kyutech.ac.jp \\ ** Department of Automatic Control and Systems Analysis, \\ Institute of Technology, Uppsala University, P.O.Box 27, \\ Uppsala, Sweden
}

\begin{abstract}
This paper is concerned with improvement of the KDI-based fault detection method so far developed by authors for nonlinear black-box systems. When modeling the system, Quasi-ARMAX model with multi-model structure is used. A fault due to unexpected change in system parameters will appear as the change of identified model. Kullback discrimination Information (KDI) can then be used as the fault detection index to evaluate the distortion in identified model. Several schemes to improve the fault detection performance are proposed, as well as the realization of a kind of fault isolation function based on a recognition approach in the model parameter space. The effectiveness of the method is verified through simulation studies on the ship propulsion system constructed for benchmark test. Copyright ${ }^{\circledR}$ 2005IFAC
\end{abstract}

Keywords: Fault detection, Kullback discrimination information, non-linear system, parameter estimation, quasi-ARMAX model, ship propulsion system

\section{INTRODUCTION}

There have been proposed many kinds of model-based fault detection method for dynamic systems (Izermann and Balle(1996)). Most of faults in dynamic systems can be represented as unexpected variations in system operating modes which are caused by changes in system configuration parameters. Based on this assumption, Kumamaru and Söderström (1986) proposed a fault detection method for linear systems. In the method, fault detection is carried out by evaluating model distortion due to a fault via Kullback discrimination information (KDI). The KDI is defined as a distortion measure for two probability density functions. It can be used for model discrimination by introducing likelihood functions corresponding to identified mod- els and explicitly analyzed under the assumption of Gaussian distribution.

On the other hand, many of object systems in real world are black-box type systems with nonlinearity. Therefore when dealing with fault detection problem in practice, an appropriate nonlinear model is required. In order to describe black-box type nonlinear systems as precisely as possible, a QuasiARMAX model was proposed (Hu, Kumamaru and Hirasawa(2001)), in which the system non-linearity is incorporated into ARMAX model parameters by using non-linear non-parametric models. However, the KDI can no longer be directly applied to evaluate the distortion of identified Quasi-ARMAX model, since the model is linear structured with respect to parameters but essentially non-linear input output model, so the corresponding likelihood function becomes non- 
Gaussian. To solve this problem, a fault detection method based on the Quasi-ARMAX model with multi-model structure was proposed, and the effectiveness was examined through simulation studies for both of many kinds of mathematical and realistic plant models.

Recently, the simulation studies of the multimodelbased fault detection method have been carried out on a ship propulsion system which was constructed as a realistic plant model for benchmark test of fault diagnosis (Zamanabadi and Blanke(1999)). The fundamental effectiveness of the method has been confirmed (Kumamaru et al(2003)). However, there have been still remained several problems concerning the deterioration of fault detection performance. In this paper, such problems to be solved are summarized and causes of the problems are investigated. Based on the investigations, several improvement schemes to fault detection are proposed. Thus the paper covers some extensions of our recent work (Kumamaru, Inoue and Iwamura (2004)). Furthermore, in this paper we will try to realize a kind of fault isolation function within the framework of our model-based fault detection approach. This can be done based on a recognition approach in the identified model parameter space. Finally simulation results applying the improved method and realized fault isolation technique are introduced with discussions.

The paper is organized as follows: in section 2, the outline of the Quasi-ARMAX model, and its multiARMAX form are briefly introduced. In section 3, the KDI analysis based on multi-model is developed in detail, together with model validation and fault detection schemes. The outline of the ship propulsion system is introduced in section 4 . In section 5, several problems concerning the deterioration of fault detection performance are introduced together with their improvement schemes. Furthermore a method to realize a fault isolation function is shown. In section 6 , simulation results based on these improvement schemes and realized fault isolation technique are summarized with discussions. Finally, section 7 is devoted to conclusions.

\section{QUASI-ARMAX MODEL}

Consider a discrete-time non-linear SISO system described by

$$
\begin{aligned}
& \mathscr{S}: y(t)=g(\varphi(t))+v(t) \\
& \varphi(t)=[-y(t-1) \cdots-y(t-n) \\
&u(t-1) \cdots u(t-m)]^{T}
\end{aligned}
$$

where $y(t)$ is the output at time $t(t=1,2 \ldots), u(t)$ the input, $v(t)$ the system disturbance, $\varphi(t)$ the regression vector and $g(\cdot)$ the unknown nonlinear function. When the system is modeled, the Quasi-ARMAX model is introduced as follows,

$$
\begin{gathered}
\mathscr{M}: A\left(q^{-1}, \phi(t)\right) y(t)=B\left(q^{-1}, \phi(t)\right) u(t) \\
+C\left(q^{-1}\right) e(t) \\
\phi(t)=\left[y(t-1) \cdots y\left(t-n^{\prime}\right)\right. \\
\left.u(t-1) \cdots u\left(t-m^{\prime}\right)\right]
\end{gathered}
$$

$$
\begin{aligned}
A\left(q^{-1}, \phi(t)\right) & =1+a_{1}(\phi(t)) q^{-1}+\cdots+a_{n}(\phi(t)) q^{-n} \\
B\left(q^{-1}, \phi(t)\right) & =b_{1}(\phi(t)) q^{-1}+\cdots+b_{m}(\phi(t)) q^{-m} \\
C\left(q^{-1}\right) & =1+c_{1} q^{-1}+\cdots+c_{l} q^{-l}
\end{aligned}
$$

where $e(t) \in \mathscr{N}\left(0, \sigma^{2}\right)$, and $q^{-1}$ is the backward shift operator. The model (2) is the same structure as a linear ARMAX model. However, $a_{i}(\phi(t))$ and $b_{i}(\phi(t))$ are the non-linear functions of $\phi(t)$ which consists of past input-output data up to $m^{\prime}$ and $n^{\prime}$, respectively. In order to describe the non-linear terms, non-linear non-parametric models (NNMs) are used as follows,

$$
\begin{aligned}
a_{i}(\phi(t))=f_{i}(\phi(t)) & (i=1, \cdots, n) \\
b_{j}(\phi(t))=f_{n+j}(\phi(t)) & (j=1, \cdots, m) \\
f_{i}(\phi(t))=\sum_{j=1}^{M} \omega_{i j} N_{f}\left(p_{j}, \phi(t)\right) & \\
& (i=1,2, \cdots n+m)
\end{aligned}
$$

where $N_{f}(\cdot)$ is the basis functions, $\omega_{i j}$ 's are the coordinate parameters to be estimated, $p_{j}$ 's are the scale and position parameters specifying the basis functions that are to be pre-assigned based on available information about the system dynamics. The model (NNMs) is equipped with flexibility for representing system nonlinearity. As an example of NNMs, Adaptive Fuzzy System (AFS) is given by

$$
f_{i}(\phi(t))=\frac{\sum_{j=1}^{M} \omega_{i j}\left(\wedge_{k=1}^{r} \mu_{A_{k}^{j}} x_{k}(t)\right)}{\sum_{j=1}^{M}\left(\wedge_{k=1}^{r} \mu_{A_{k}^{j}} x_{k}(t)\right)}
$$

where $x_{k}(t)$ is the $k$-th element of $\phi(t) . M$ and $r$ are the number of fuzzy rules and of inputs to AFS, respectively. $\mu_{A_{k}^{j}}$ is the membership function corresponding to a $j$-th fuzzy rule and $\wedge_{k=1}^{r}$ denotes minimum operator. Using (3), (4) in (2), the Quasi-ARMAX model (2) can be transformed into the multi-model form,

$$
\begin{aligned}
\mathscr{M}\left(\Omega_{e}\right) & : \\
y(t) & =\sum_{j=1}^{M} \varphi(t)^{T} \Omega_{j} N_{f}\left(p_{j}, \phi(t)\right)+C\left(q^{-1}\right) e(t) \\
& =\sum_{j=1}^{M}\left(\varphi_{e}(t)^{T} \Omega_{e j}+e(t)\right) N_{f}\left(p_{j}, \phi(t)\right) \quad(5) \\
\varphi_{e}(t) & =\left[\begin{array}{lll}
\varphi(t)^{T} & e(t-1) \cdots e(t-l)
\end{array}\right]^{T} \\
\Omega_{j} & =\left[\begin{array}{lll}
\omega_{1 j} & \cdots & \omega_{(n+m) j}
\end{array}\right]^{T} \\
\Omega_{e j} & =\left[\begin{array}{lll}
\Omega_{j}^{T} c_{1} & \cdots & c_{l}
\end{array}\right]^{T} \\
\Omega_{e} & =\left[\begin{array}{lll}
\Omega_{e 1}^{T} & \cdots & \Omega_{e M}^{T}
\end{array}\right]^{T} \quad(j=1, \cdots, M)
\end{aligned}
$$


The parameter estimation for (5) can be performed by existing method of recursive identification , e.g., the prediction error method (Ljung and Söderström(1983)) When the basis function have compact supports, the identified model has a multi-ARMAX model structure interpolated by the basis functions, which consists of $M$ local identified models

$$
\begin{gathered}
\mathscr{M}\left(\hat{\Omega}_{e j}\right): A\left(q^{-1}: \hat{\Omega}_{e j}\right) z_{j}(t)=B\left(q^{-1}: \hat{\Omega}_{e j}\right) u(t) \\
+C\left(q^{-1}: \hat{\Omega}_{e j}\right) \varepsilon(t) \\
A\left(q^{-1}: \hat{\Omega}_{e j}\right)=1+\hat{\omega}_{1 j} q^{-1}+\cdots+\hat{\omega}_{n j} q^{-n} \\
B\left(q^{-1}: \hat{\Omega}_{e j}\right)=\hat{\omega}_{(n+1) j} q^{-1}+\cdots+\hat{\omega}_{(n+m) j} q^{-m} \\
C\left(q^{-1}: \hat{\Omega}_{e j}\right)=1+\hat{c}_{1}+\cdots+\hat{c}_{l} q^{-l} \\
(j=1, \cdots M)
\end{gathered}
$$

where $\varepsilon(t)$ can be considered to to be $\varepsilon(t) \in \mathscr{N}\left(0, \hat{\sigma}^{2}\right)$, and the symbol $\hat{\bullet}$ means the estimated value and $z_{j}(t)$ stands for the output variable of the $j$-th local identified model.

\section{KULLBACK DISCRIMINATION INFORMATION (KDI)}

When a fault has occurred in the system, the effect will appear as a change in identified model. We can thus detect the fault by discriminating the difference between two models which are identified in disjunctive intervals. Such a difference can be evaluated by using KDI. This is basic idea of our fault detection scheme based on the KDI. The KDI, a distortion measure of two probability density functions, was developed as an effective index to detect the fault in linear dynamics system with Gaussian stochastic properties (Kumamaru and Söderström(1986)). As is mentioned in section 2, when the objective system is a black-box type non-linear system, the Quasi-ARMAX model with multi-model form (5) is used to identify the system. And the fault effect will appear as a change in estimated parameter $\hat{\Omega}_{e}$. However the KDI can not be applied to discriminate the identified multi-model, because the model is really a non-linear one. In order to solve this problem, the KDI is used to evaluate the difference in each identified local linear ARMAX model described by (6), then the overall difference in the identified multi-ARMAX model $\mathscr{M}\left(\hat{\Omega}_{e}\right)$ can be estimated from these local model differences.

\subsection{KDI analysis}

Assume that data from the system are available from two disjunctive time intervals $I_{1}$ and $I_{2}$ with number of data set $N_{1}, N_{2}$, respectively. Perform identification for the multi-ARMAX model (5) using data obtained from $I_{1}$ and $I_{2}$, and denote by $\hat{\Omega}_{e j i}(j=1, \cdots, M)$, $(i=1,2)$ the estimates of $j$-th local model parameters.
We thus have identified local linear ARMAX models $\mathscr{M}\left(\hat{\Omega}_{e j 1}\right), \mathscr{M}\left(\hat{\Omega}_{e j 2}\right)(j=1, \cdots, M)$. Introduce the KDI defined for the likelihood functions corresponding to these two models

$$
\begin{aligned}
I_{N_{1}}[1,2](j)= & \int p\left(Z_{N_{1}}^{(j)} \mid \hat{\Omega}_{e j 1}, U_{N_{1}-1}\right) . \\
& \ln \frac{p\left(Z_{N_{1}}^{(j)} \mid \hat{\Omega}_{e j 1}, U_{N_{1}-1}\right)}{p\left(Z_{N_{1}}^{(j)} \mid \hat{\Omega}_{e 2 j}, U_{N_{1}-1}\right)} d Z_{N_{1}}^{(j)}
\end{aligned}
$$

where $U_{N_{1}-1}=\left[u(1), \cdots, u\left(N_{1}-1\right)\right]^{T}$ are input data sets in the interval $I_{1}$, and $Z_{N_{1}}^{(j)}=\left[z_{j}(1), \cdots, z_{j}\left(N_{1}\right)\right]^{T}$ are augmented output variables of $j$-th local identified model. The index in (7) hence indicates how well the model using $\hat{\Omega}_{e j 2}$ describes the data in the interval $I_{1}$. Due to the Gaussian assumption on $\varepsilon(t)$ in (6), the likelihood functions corresponding to identified local linear ARMAX models are also Gaussian distributed. Therefore, using Bayesian rule, (7) can be analyzed into an explicit form, by which the KDI's for each identified local linear ARMAX model can be evaluated in a feasible way for finite but fairly large data set. The result of such an analysis is:

$$
\begin{array}{r}
I_{N_{1}}[1,2](j)=I_{N_{1}}^{(1)}[1,2](j)+I_{N_{1}}^{(2)}[1,2](j) \\
+I_{N_{1}}^{(3)}[1,2](j)
\end{array}
$$

where each component of $I_{N_{1}}[1,2](j)$ is given by

$$
\begin{aligned}
& I_{N_{1}}^{(1)}[1,2](j)=\frac{N_{1}}{2}\left[\left(\hat{\sigma}_{1} / \hat{\sigma}_{2}-1\right)-\ln \left(\hat{\sigma}_{1} / \hat{\sigma}_{2}\right)\right] \\
& I_{N_{1}}^{(2)}[1,2](j)=\frac{1}{2} \sum_{k=0}^{N_{1}-1} \|\left(H_{2}^{(j)}\right)^{-1} \cdot \\
& \quad\left(G_{1}^{(j)}-G_{2}^{(j)}\right) u(k+1) \|_{\hat{\sigma}_{2}^{-2}}^{2} \\
& I_{N_{1}}^{(3)}[1,2](j) \quad(j=1, \cdots M) \\
& =\frac{N_{1}}{2}\left[\frac{\hat{\sigma}_{2}^{-2}}{2 \pi i} \oint\left\{\left(H_{2}^{(j)}(z)\right)^{-1} H_{1}^{(j)}(z)-1\right\}\right. \\
& \left.\hat{\sigma}_{1}^{2}\left\{H_{1}^{(j)}\left(z^{-1}\right)\left(H_{2}^{(j)}\left(z^{-1}\right)\right)^{-1}-1\right\} \frac{d z}{z}\right]
\end{aligned}
$$

and each simplified variable is defined by

$$
\begin{aligned}
G_{i}^{(j)} & =B\left(q^{-1}: \hat{\Omega}_{e j i}\right) / A\left(q^{-1}: \hat{\Omega}_{e j i}\right) \\
H_{i}^{(j)} & =C\left(q^{-1}: \hat{\Omega}_{e j i}\right) / A\left(q^{-1}: \hat{\Omega}_{e j i}\right) \\
H_{i}^{(j)}(z) & =H\left(q^{-1}: \hat{\Omega}_{e j i}\right) / q^{-1}=z \\
& (i=1,2),(j=1, \cdots M)
\end{aligned}
$$

The difference measure for the identified multiARMAX models are estimated from $I_{N_{1}}[1,2](j)$ in (8), by weighting average of them over the number of multi-models $M$. 


$$
I_{F D}=\frac{1}{M} \sum_{j=1}^{M} W(j) I_{N_{1}}[1,2](j)
$$

where $W(j)$ 's are weighting factors. It is expected that an appropriate choice of weighting factors in (12) will make the index more sensitive to the change of identified multi-ARMAX model. Considering the structure of the multi-model (5) interpolated by the basis functions, we will set the weights by

$$
W(j)=\frac{1}{N_{1}} \sum_{t=1}^{N_{1}} N_{f}\left(p_{j}, \phi(t)\right)
$$

In this way, the index $I_{F D}$ can be used to evaluate the difference between two identified Quasi-ARMAX models with multi-model form. Such a model discrimination based on the KDI can be applied to two problems, model validation and fault detection problems.

\subsection{Model validation}

In the model-based approach to fault detection, it is important task to test the adequateness of the model, this is so called "model validation". Let us assume that the object system is time-invariant under the normal operation. Then, when the model structure is appropriately chosen, the difference between two identified models obtained from disjunctive intervals $I_{1}$ and $I_{2}$ under the normal mode may be comparatively small. On the other hand, if the model structure is not good enough to describe the system, the model parameter estimates will fluctuate strongly depending on the identification conditions. Therefore, the difference measure $I_{F D}$ in (12) for two identified models can also be used as the index for model validation.

\subsection{Fault detection scheme}

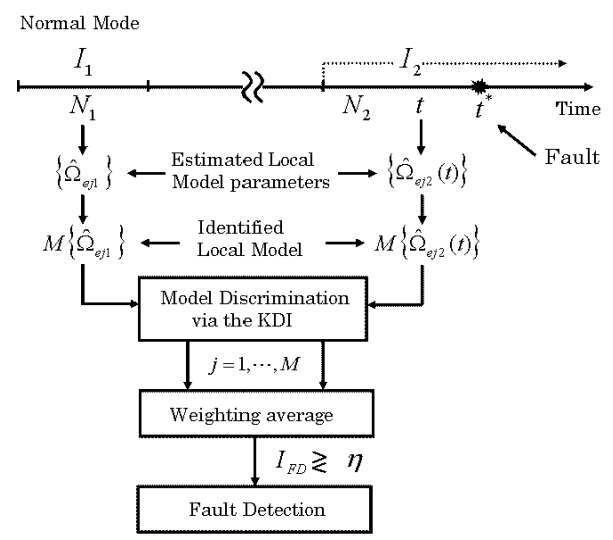

Fig. 1. Fault detection scheme

Fig.1 explains the procedure of KDI-based fault detection. Assume that the data are available from two disjunctive intervals $I_{1}$ and $I_{2}$ with number of data sets $N_{1}$ and $N_{2}$, respectively, where the system is under normal mode in the interval $I_{1}$ and is monitored for fault detection in the interval $I_{2}$. Perform the identification based on the Quasi-ARMAX model with multi-model form. Denote the identified model obtained in $I_{1}$ by $\mathscr{M}\left\{\hat{\Omega}_{e j 1}\right\}$, and the on-line identified model obtained in $I_{2}$ by $\mathscr{M}\left\{\hat{\Omega}_{e j 2}(t)\right\}$, respectively. The differences between two identified local ARMAX models are evaluated by the KDI, $I_{N_{1}}[1,2](j)$ in (8) and the differences measure $I_{F D}$ for the two identified Quasi-ARMAX models can be obtained from weighting average of these KDI's. Then the fault detection is executed online based on the threshold decision approach by using the detection index $I_{F D}$.

\section{SHIP-PROPULSION SYSTEM}

As the plant to be diagnosed, a ship-propulsion system with one engine and one propeller is considered. This system-model has been constructed as the model for benchmark test of fault diagnosis (Zamanabadi and Blamke(1999)). The schematic diagram of this system is shown in Fig.2.

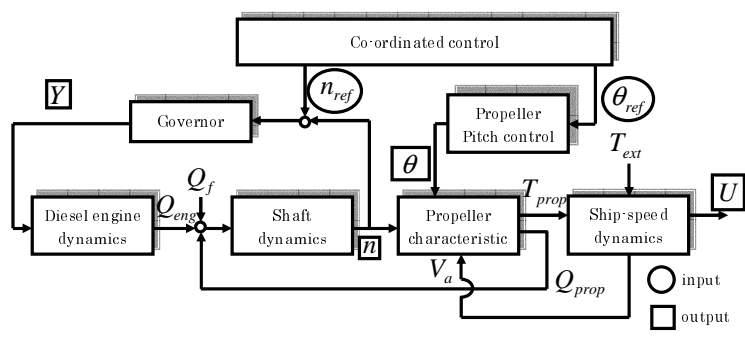

Fig. 2. Schematic diagram of the ship-propulsion system

Main components/subsystems of the system are 1) propeller pitch control system, 2) governor, 3) diesel engine, 4) shaft dynamics, 5) propeller characteristics, and 6) ship speed dynamics. This system is 6-ordered nonlinear system with two inputs and four measurable outputs (See Fig.2). They are as follows: two inputs are 1) the shaft speed reference $n_{\text {ref }}$ and 2) the propeller pitch reference $\theta_{\text {ref }}$, four outputs are 1) the diesel engine shaft speed $n, 2)$ propeller pitch position $\theta, 3)$ the fuel index $Y$ and 4) the ship speed $U$.

In the figure, $Q_{f}$ and $T_{\text {ext }}$ denotes the friction torque and the external thrust force, respectively. Disturbance factors such as $v_{n}, v_{\theta}, v_{Y}$ and $v_{U}$ are considered in $n$, $\theta, Y$ and $U$, respectively as the Gaussian distributed variables with zero mean. The friction torque is random valued so it is treated as a disturbance. The ship plant model can realize many kinds of fault mode including both of dynamics and sensor faults.

\section{IMPROVEMENT SCHEMES}

So far, the fundamental effectiveness of the KDIbased fault detection method has been confirmed through simulation studies on the ship-propulsion system (Kumamaru et al(2003)). However in this method 
there have been still remained problems concerning performance deterioration. In this section, causes of such problems are investigated and their improvement schemes are proposed.

The first problem is that the convergence rate of identifying the Quasi-ARMAX model is very slow compared to the identification of linear model. This is because that the Quasi-ARMAX model has large number of parameters to be estimated (see $\mathscr{M}\left(\Omega_{e}\right)$ in eq.(5)). Therefore in order to obtain well converged identified model with accuracy enough to be used as the reference model, fairly large amount of data set is required in the interval $I_{1}$. To solve this problem we considered a cyclic re-utilization of given data set with reasonable amount and its effect could be examined by the model validation technique using the KDI.

The second problem is that the KDI value occasionally increases abruptly under the normal plant operation. This means that the identified model in the interval $I_{2}$ has changed from the normal model although the plant has no fault. After the careful investigation, it was found that such phenomena were caused by jump change of estimated model parameter in the recursive identification procedure, where input-output data corresponding to rare case of plant operation mode were obtained. To avoid these undesirable phenomena, we tried to remove such rare case data from the identification algorithm, based on the pre-evaluation of a data utility factor which is calculated from basis function in a similar way to (13).

The third problem is that in the beginning of fault detection interval $I_{2}$ where any fault has not yet occurred, the model parameter estimates may change from those of reference model obtained at final stage of the interval $I_{1}$. This is because that the Quasi-ARMAX model is a kind of state dependent type model, so the parameter estimation is strongly affected by plant inputoutput data used in the identification. Therefore, in order to start successfully the fault detection procedure, some continuity of input data sequence is required between last part of the interval $I_{1}$ and first part of the interval $I_{2}$.

Furthermore, we tried to realize a kind of fault isolation function within the framework of the KDI-based fault detection method. This might be achieved based on the recognition approach in the identified QuasiARMAX model parameter space, where a feature space for fault recognition is pre-constructed based on the knowledge about the primitive fault modes.

These improvement schemes are actively considered in the following simulation studies.

\section{SIMULATION STUDIES}

\subsection{Quasi-ARMAX modeling}

For convenience's sake, the part of "Governor - Diesel Engine - Propeller Shaft" is selected as the objective system for fault detection. To describe the system, let us consider the following Quasi-ARMAX model;

$$
\begin{array}{r}
A\left(q^{-1}, \phi(t)\right) y(t)=B_{1}\left(q^{-1}, \phi(t)\right) u_{1}(t) \\
+B_{2}\left(q^{-1}\right) u_{2}(t)+C\left(q^{-1}\right) e(t)
\end{array}
$$

Where the input variable $u_{1}$ is the shaft speed reference $n_{\text {ref }}$ and the torque $Q_{\text {prop }}$ developed from propeller dynamics stands for another input variable $u_{2}$, while the output variable $y$ is the measurement of the diesel engine shaft speed $n$. Note that the nonlinear elements included in the system are saturation and delay type ones. There are several factors specifying the model structure of the Quasi-ARMAX model. They are the model orders $n, m_{1}, m_{2}, l$, the components of the vector $\phi$, i.e. fuzzy variables, and the number of fuzzy rule $M$. These factors are determined appropriately according to the model validation procedure as is mentioned in Section 3.2. Such the model validation test has been executed during the experimental identification procedure under the normal mode in the interval $I_{1}$ with data set 80,000 . As to the data acquisition for the identification, the object system is driven by a given input sequence $u_{1}(t)$ with rectangular form which randomly takes four different vales, and resulting output sequence $y(t)$ is observed, while another input $u_{2}(t)$ is treated as known valued internal variable of the ship propulsion system. As the result, we have had the following model structure:

$$
\begin{aligned}
& \text { - model order: } n=3, m_{1}=4, m_{2}=2, l=1 \\
& \text { - number of fuzzy rule : } M=16 \\
& \cdot \text { fuzzy variable : } \phi(t)=\left[y(t-1) u_{1}(t-1)\right]^{T} \\
& \qquad\left(n^{\prime}=1, m^{\prime}=1\right)
\end{aligned}
$$

We will use the identified model obtained at the last stage of interval $I_{1}$ as the reference model for the fault detection.

\subsection{Simulation Results of Fault Detection}

Based on the several improvement schemes proposed in the previous section, simulations studies on fault detection have been carried out for many kinds of dynamics fault under both of deterministic and stochastic situations. Here in the stochastic situation, the plant suffers from disturbances $v_{n}, v_{\theta}, v_{Y}, v_{U}$ and $Q_{f}$. As the fault modes, abrupt changes at a time $t^{*}$ in the plant parameters, e.g., engine gain $k_{y}$, shaft moment of inertia $I_{m}$, governor gain $k_{r}$ and fuel index $Y$ are considered.

Among many simulation results, the result for a fault in $k_{y}$ under the deterministic situation is introduced here. Where the fault occurrence time $t^{*}$ is taken to be $1000 t h$ step in the interval $I_{2}$ with number of data set $N_{2}=2000$ and the degree of the parameter change is specified to $-20 \%$ of its rating value.

The result is shown in Fig. 3 as the evaluated KDI sequences, where the KDI in (8) is calculated using input data sets $U_{N_{1}-1}$ in the interval $I_{1}$ with $N_{1}=100$. 


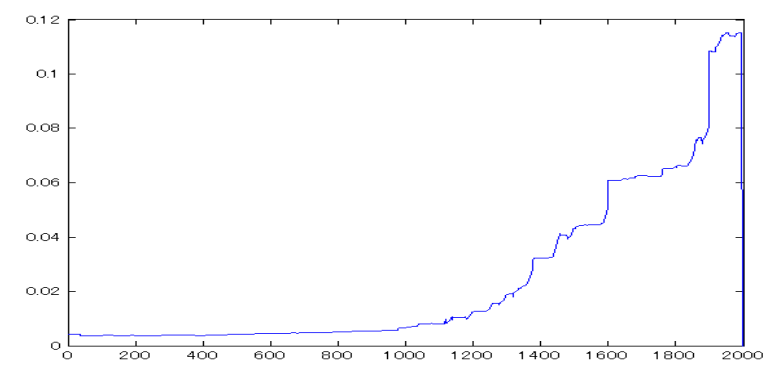

Fig. 3. Evaluated KDI under deterministic situation

Table 1. Fault Detection for Other Modes

\begin{tabular}{c|c|c|c|c}
\hline \hline fault modes $\backslash$ fault degree & $30 \%$ & $20 \%$ & $10 \%$ & $5 \%$ \\
\hline engine gain $k_{y}$ & $\bigcirc$ & $\bigcirc$ & $\triangle$ & $\times$ \\
\hline moment of inertia $I_{m}$ & $\bigcirc$ & $\bigcirc$ & $\triangle$ & $\times$ \\
\hline governor gain $k_{r}$ & $\bigcirc$ & $\bigcirc$ & $\bigcirc$ & $\triangle$ \\
\hline fuel index $Y$ & $\bigcirc$ & $\bigcirc$ & $\triangle$ & $\times$ \\
\hline
\end{tabular}

Table 2. Fault Isolation

\begin{tabular}{c|ccccc}
\hline \hline Failed Factor $\backslash$ Isolated Fault & $k_{y}$ & $I_{m}$ & $k_{r}$ & $Y$ & Normal \\
\hline engine gain $k_{y}$ & 60 & 0 & 0 & 40 & 0 \\
moment of inertia $I_{m}$ & 0 & 100 & 0 & 0 & 0 \\
governor gain $k_{r}$ & 0 & 0 & 96 & 0 & 4 \\
fuel index $Y$ & 56 & 0 & 0 & 44 & 0 \\
no fault & 0 & 0 & 0 & 0 & 100 \\
\hline
\end{tabular}

It can be seen that the fault occurrence can definitely be detected by monitoring the index of KDI. On the other hand, the adequateness of the identified QuasiARMAX model with structure specified in the simulation can also be confirmed from the result shown in Fig. 3, since the model distortion measure KDI evaluated under the normal mode (i.e., until $t^{*}=1000$ ) is kept to be small value. This means that the system parameters do not change, i.e. there is no fault. For other fault modes with different fault degrees are summarized in Table 1 as the evaluation indices of the fault detectability, where the symbol $\bigcirc, \triangle$ and $\times$ denotes the degree of detectability, i.e., possible, fairly possible and impossible, respectively.

On the other hand, in the stochastic situation the fault detection is still possible within relatively weak disturbance, although the KDI evaluated under normal mode becomes more larger than the deterministic case, and the fault detection ability may degrade as disturbances become larger and larger.

\subsection{Simulation Results of Fault Isolation}

It is expected that the fault mode occurred in the system will be reflected more or less in the estimated parameter of the Quasi-ARMAX model. This suggests us some possibility to realize a fault isolation function within the framework of our model-based approach to fault detection. We tried a fault recognition in the feature space which was constructed by model parameters based on knowledge about typical fault modes. In our simulation ( the model in (14)), the parameter to be estimated consists of $\left(n+m_{1}\right) M+m_{2}+l=115$ elements. For each element, absolute value of the difference between two estimated parameters obtained at the end of intervals $I_{1}$ and $I_{2}$ was used as the feature index in the 115-dimensional feature space. The result of fault isolation is shown in Table 2, as the percentage recognition rate among the 25 samples for each fault mode. It can be seen that each fault mode can be isolated with high precision, except for the faults in the engine gain and the fuel index. The difficulty of the isolation between $k_{y}$ and $Y$ results from the peculiar system configuration.

\section{CONCLUSIONS}

In this paper, a fault detection method using QuasiARMAX model with multi-model form was proposed, and simulation studies on the ship propulsion system were carried out for various dynamics fault modes under both of deterministic and stochastic situations. In particular, several problems concerning the deterioration of fault detection performance were investigated, and their improvement schemes were proposed, as well as a technique to realize the fault isolation function. As the result, the effectiveness of the method and its applicability to realistic plant have been confirmed with more higher confidence compared to previous our works.

\section{REFERENCES}

Hu,J.,K.Kumamaru and K.Hirasawa(2001).

A Quasi-ARMAX approach to modelling of nonlinear systems. Interna. J. Control,

74-18,1754-1766.

Izadi-Zamanabadi, R. and M.Blanke(1999). Ship propulsion system as a benchmark for fault tolerant control. Control Eng. Practice, 7-3, 227-239.

Izermann,R. and P.Balle(1996). Trends in the application of model based fault detection and diagnosis of technical processes. In: Proc of 13th World Congress of IFAC, Vol.N,1-12.

Kumamaru,K. and T.Söderström(1986). Fault detection and model validation using index of Kullback discrimination information. Trans. of the Society of Instrument and Control Engineers, 22-10,11351140.

Kumamaru,K., K.Inoue, Y.Hosoyamada and T.Söderström (2003). Fault detection of non-linear systems based on multi-form quasi-ARMAX modeling and its application to the ship benchmark. In:Proc. of the 13th IFAC Symp. on System Identification.

Kumamaru,K., K.Inoue and T.Iwamura (2004). Improved approach to KDI-based fault detection for non-linear black-box systems.In :Proc. of the SICE Annual Conference 2004 in Sapporo, 927-932.

Ljung,L. and T.Söderström(1983). Theory and Practice of Recursive Identification. The MIT Press, Cambridge,Mass.. 